Note

\title{
Beef Tallow Diet Decreases Uncoupling Protein Content in the Brown Adipose Tissue of Rats
}

\author{
Tatsuhiro MAtsuo, ${ }^{1, *}$ Maki Komuro, ${ }^{2}$ and Masashige SuZukI ${ }^{2}$ \\ ${ }^{1}$ Division of Nutrition and Biochemistry, Sanyo Women's College, \\ Hatsukaichi 738, Japan \\ ${ }^{2}$ Institute of Health and Sport Sciences, University of Tsukuba, \\ Tsukuba 305, Japan
}

(Received June 11, 1996)

\begin{abstract}
Summary The effects of dietary fats consisting of different fatty acids on the content of mitochondrial uncoupling protein in the interscapular brown adipose tissue were studied in rats. Sprague-Dawley male rats were meal-fed an isoenergetic diet based on either beef tallow or safflower oil for nine weeks. The gain in body weight during the experimental period did not differ between the two dietary groups. The weight of the brown adipose tissue was similar in the two dietary groups, whereas the weight of the abdominal white adipose tissue was larger in rats fed the beef tallow diet. The content of mitochondrial uncoupling protein in brown adipose tissue was lower in the beef tallow diet group than in the safflower oil diet group without differing mitochondrial mass between the two dietary groups. These results suggest that, in rats, a beef tallow diet reduces the content of uncoupling protein in brown adipose tissue, resulting in lower diet-induced thermogenesis as compared to a safflower oil diet.
\end{abstract}

Key Words beef tallow, safflower oil, diet-induced thermogenesis, uncoupling protein, brown adipose tissue, rat

Diet-induced thermogenesis (DIT) in brown adipose tissue (BAT) has become recognized as a significant component of energy expenditure, especially in rodents: a component that may tip the energy balance toward leanness or obesity (1). Since it was observed that certain types of diets, so-called "cafeteria diets," could stimulate DIT, it has been of interest to analyze to what extent specific diet constituents themselves may possess in terms of properties to enhance the thermogenic effect (2).

On the other hand, it has been reported that DIT is affected by dietary fatty-acid composition $(3,4)$. We recently demonstrated that DIT and the fat oxidation rate were lower in rats fed a beef tallow diet than in rats fed a safflower oil diet. This resulted in increased body fat accumulation in the beef tallow diet

\footnotetext{
* To whom correspondence should be addressed.
} 
group when rats were fed isoenergetic diets (45\% of energy as fat) based on beef tallow-enriched saturated fatty acid or safflower oil-enriched $n-6$ polyunsaturated fatty acid for four months (3). Moreover, rats fed a beef tallow diet showed lower DIT by the decrease of sympathetic activity in the interscapular BAT (4).

DIT in rats is mainly caused by a proton conductance pathway in BAT mitochondria that allows protons to 'leak' back across the inner mitochondrial membrane without obligatory ATP synthesis (5). This pathway exists in proteins of molecular weight $32 \mathrm{kDa}$ in the inner mitochondrial membrane of BAT (5). These proteins are called thermogenin or uncoupling protein (UCPs), and a higher thermogenic capacity of the whole animal occurs due to UCP (5). It is known that rats with hypothalamic obesity (rats with ventromedial hypothalamus lesions) or genetically obese rats are unable to activate DIT. Vander Tuig et al. suggested that the thermogenic capacity of UCP in BAT was lower in rats with hypothalamic obesity than in control rats (6). Other researchers reported that BAT in the genetically obese Zucker rat was in a quiescent and atrophied state with progressive reduction in the mitochondrial UCP content (7) and mRNA level (8), as well as having low sympathetic activity (9) in its BAT. From these points of view, we speculated that decreased DIT induced by a beef tallow diet is the result of reducing the UCP content in BAT accompanied by decreasing sympathetic activity in BAT. In this study, we investigated the UCP content in the interscapular BAT of rats fed a beef tallow diet or a safflower oil diet for nine weeks.

\section{Experimental}

All procedures involving animals were approved by the Experimental Animal Care Committee of the University of Tsukuba.

Animal care and experimental design. Forty-two male Sprague-Dawley rats (5-weeks-old) were obtained from CLEA Japan (Tokyo). One-half of the animals were fed a safflower oil diet and the other half were fed a beef tallow diet. The compositions of both diets have been described previously $(3,4)$. Both diets provided 45,35 and $20 \%$ of the energy as fat, carbohydrate and protein, respectively. The available metabolizable energy was $19.7 \mathrm{~kJ} / \mathrm{g}$ for the safflower oil diet and $18.4 \mathrm{~kJ} / \mathrm{g}$ for the beef tallow diet. The fatty-acid compositions of safflower oil and beef tallow have been described previously $(3,4)$. Beef tallow consists of $44 \%$ oleic, $27 \%$ palmitic and $18 \%$ stearic acids, and safflower oil consists of $79 \%$ linoleic acid. The animals were individually caged at $22 \pm 2{ }^{\circ} \mathrm{C}$, with light from 0700 to $1900 \mathrm{~h}$. Each group of rats was meal-fed the diet at $0800-0900 \mathrm{~h}$ and $2000-2100 \mathrm{~h}$, and was given free access to water for nine weeks. Both groups of rats were offered the appropriate diet in amounts such that the two groups consumed equal metabolizable energy during the experimental period. The meal-feeding method was used to adjust the energy intake between the two dietary groups. Under meal-feeding conditions, feeding one meal (within $2 \mathrm{~h}$ ) a day decreased the food intake of the animals; however, feeding two meals a day, as in this study, minimized this effect of meal-feeding. The food consumption of rats calculated at $21.3 \mathrm{MJ} / 9$ weeks was 
approximately the maximal amount of diet that rats could consume under the meal-feeding conditions. On the final day, the rats of both diet group were decapitated at $0800 \mathrm{~h}$. The interscapular BAT and abdominal adipose tissues (epididymal, perirenal and mesenteric) were removed and weighed, and then the BAT was immediately frozen in liquid nitrogen and stored at $-80^{\circ} \mathrm{C}$ until analysis.

Preparation of mitochondria. BAT mitochondria was prepared by a method described previously (10). Briefly, BAT was homogenized in $5 \mathrm{ml}$ of $0.01 \mathrm{M}$ phosphate-buffered saline (PBS) $(\mathrm{pH} 7.2)$ and then centrifuged at $700 \times g$ for 10 min. The supernatant below the fat layer was sucked out carefully and then centrifuged at $6,000 \times g$ for $10 \mathrm{~min}$. The pellet was resuspended in $0.01 \mathrm{M}$ PBS (pH 7.2). The mitochondrial fraction was rinsed and centrifuged three times at 6,000 $\times g$ for $10 \mathrm{~min}$. The final pellet was resuspended in PBS at a protein concentration of $2 \mathrm{mg} / \mathrm{ml}$ and the mitochondrial fraction was immediately analyzed. Protein was determined by the Bradford method (11).

Immunoassay of UCP and mitochondria antigen. The immunological determination of UCP and mitochondria antigen (MW, $65 \mathrm{kDa}$ ) was made by Western blot analysis. Rabbit anti-rat UCP was donated by Dr. Kawada, Department of Food Science and Technology, Faculty of Agriculture, Kyoto University. The monoclonal antibody to mitochondria was purchased from Biogenesis Ltd. (Poole, UK). Protein from the BAT, $2 \mathrm{mg} / \mathrm{ml}$, was subjected to SDS-polyacrylamide gel electrophoresis in 11\% polyacrylamide slab gels according to the method of Laemmli (12). Following electrophoresis, the proteins were electrophoretically transferred to a nitrocellulose membrane using the method of Burnette (13). The immunoreactivity of the UCPs and mitochondrial antigens was detected by the ECL Western blotting system (Amersham, Buckinghamshire, UK).

Statistical analysis. The significant difference between the two dietary groups was tested by Student's $t$-test.

\section{Results and discussion}

The gain in body weight during the 9-week experimental period was not significantly different between the two dietary groups (Table 1). The BAT weight was not different between the groups, whereas the weight of the abdominal adipose tissue was significantly higher in the beef tallow diet group as compared to the safflower oil diet group $(p<0.05)$ (Table 1). These results were similar to those reported previously $(3,4)$. The lipid and protein contents in the BAT were not different between the groups.

The UCP content in the BAT was lower in the beef tallow diet group than in the safflower oil diet group $(p<0.01)$ (Fig. 1A), whereas the mitochondrial antigen content was not significantly different between the two dietary groups (Fig. 1B).

The present results demonstrated that, compared to a safflower oil diet, a beef tallow diet decreases the BAT UCP content without altering the BAT mitochondrial mass. Because both rat groups consumed diets with the same amount of metabolizable energy throughout the experimental period, the difference in the 
Table 1. Effect of the dietary fats on body weight and tissue weights in rats. ${ }^{1}$

\begin{tabular}{lcc}
\hline & \multicolumn{2}{c}{ Diet group } \\
\cline { 2 - 3 } & Safflower oil & Beef tallow \\
$n=21$ & $n=21$ \\
\hline Body weight & & \\
Initial (g) & $136 \pm 5$ & $133 \pm 4$ \\
Final (g) & $438 \pm 20$ & $428 \pm 14$ \\
Gain (g) & $302 \pm 18$ & $295 \pm 15$ \\
Brown adipose tissue & & \\
Weight (mg) & $523 \pm 64$ & $522 \pm 113$ \\
Fat (mg) & $284 \pm 34$ & $276 \pm 32$ \\
Protein (mg) & $84.4 \pm 1.2$ & $87.6 \pm 3.2$ \\
Abdominal adipose tissues ${ }^{2}$ & & \\
(g) & $21.5 \pm 4.3$ & $23.9 \pm 2.7^{*}$ \\
(\%) & $4.9 \pm 1.0$ & $5.7 \pm 0.7^{* *}$ \\
\hline
\end{tabular}

${ }^{1}$ Values are $\mathbf{M} \pm \mathbf{S E M}$ for 21 rats except for the protein content of brown adipose tissue $(17$ rats). Statistically significant difference $(* p<0.05, * * p<0.01)$ from the safflower oil diet group (Student's $t$-test).

${ }^{2}$ Abdominal adipose tissues (epididymal, perirenal and mesenteric).
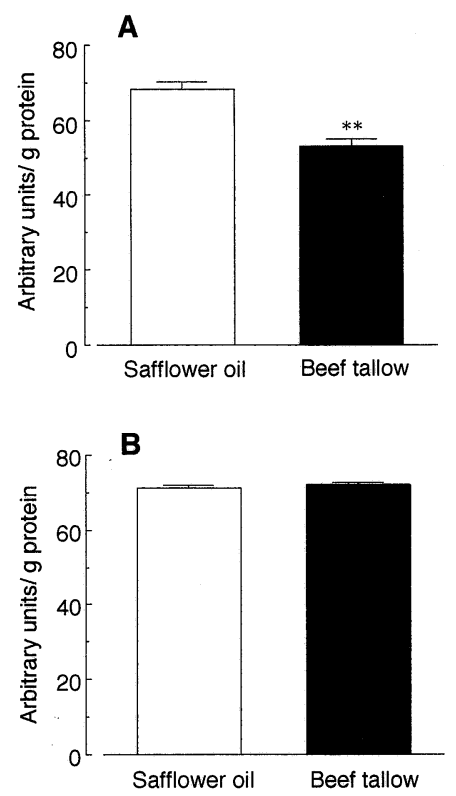

Fig. 1. The relative content of mitochondrial UCP (A) and mitochondrial antigens (B) in the BAT of rats fed beef tallow diet or safflower oil diets. Values are $\mathbf{M} \pm$ SEM for 10 rats. ${ }^{* *}$ Statistically significant difference from the safflower oil diet group. Differences with $p<0.01$ (Student's $t$-test) were considered significant. 
BAT UCP content between the two dietary groups was ascribed to different dietary fats.

DIT in the BAT was regulated by the sympathetic nervous system, especially via $\beta$-adrenergic receptors. $\beta$-Adrenergic receptors coupled with adenylate cyclase in the plasma membrane of brown adipocyte, and when norepinephrine binds to $\beta$-adrenergic receptors, adenylate cyclase is activated (14). Adenylate cyclase catalyzes the production of cyclic AMP, an intracellular messenger, which in turn activates the hormone sensitive lipase in BAT. Free fatty acids released from triacylglycerol by the hormone sensitive lipase are oxidated in BAT mitochondria. It has been suggested that the triacylglycerol turnover (fat catabolism and anabolism) in BAT is accelerated by electrical stimulation of the ventromedial hypothalamus nucleus, known as a main regulatory center in the sympathetic nervous system (15). The reduction of sympathetic activity in BAT decreases the triacylglycerol turnover and fatty acid oxidation in BAT mitochondria, resulting in reduced DIT. In this study, the lipid content in BAT was not different between the two dietary groups, whereas the triacylglycerol turnover and fatty acid oxidation in BAT mitochondria may have been decreased in the beef tallow diet group as compared to the safflower oil diet group, because sympathetic activity in BAT is decreased in the former (4). From these points of view, the reduction of UCP content in the beef tallow diet group may be related to the decrease in fatty-acid supply to BAT mitochondria.

On the other hand, Bouillaud et al. (16) reported that norepinephrine increased the level of UCP mRNA, consequently increasing the UCP content. Lower sympathetic activity in the BAT of rats fed a beef tallow diet may reduce the level of UCP mRNA, thus resulting in the reduction of UCP content. Sadurskis et al. (17) demonstrated that the metabolic response to norepinephrine and the amount of UCP were lower in mice fed a low-polyunsaturated fatty-acid (PUFA) diet than those fed a high-PUFA diet (a two-week experimental diet). Our present findings support those results.

In conclusion, this study demonstrated that dietary saturated fats reduce the UCP content in BAT without altering the mitochondrial mass. Lower DIT in rats fed a beef tallow diet may be caused by the reduction of UCP content in BAT.

We thank Dr. Teruo Kawada, Department of Food Science and Technology, Faculty of Agriculture, Kyoto University, for donating rabbit anti-rat UCP. We thank Dr. Hiroshi Sumida, Sanyo Women's College, and Dr. Mineo Yasuda, Hiroshima University, School of Medicine, for their invaluable assistance with various aspects of our studies. We also thank Ms. Ryoko Ueki and Ms. Yuka Suyama for their technical assistance. This study was supported by a Grant-in-Aid for Encouragement of Young Scientists from the Ministry of Education, Science, Sports and Culture of Japan. 


\section{REFERENCES}

1) Rothwell, N. J., and Stock, M. J. (1983): Diet-induced thermogenesis, in Mammalian Thermogenesis, ed. by Giradier, L., and Stock, M. J., Chapman and Hall, London, pp. 208-233.

2) Himms-Hargen, J. (1990): Brown adipose tissue thermogenesis: role in thermoregulation, energy regulation and obesity, in Thermoregulation, ed. by Schonbaum, E., and Lomax, P., Pergamon Press, New York, pp. 324-414.

3) Shimomura, Y., Tamura, T., and Suzuki, M. (1990): Less body fat accumulation in rats fed a safflower oil diet than in rats fed a beef tallow diet. J. Nutr., 120, 1291-1296.

4) Matsuo, T., Shimomura, Y., Saitoh, S., Tokuyama, K., Takeuchi, H., and Suzuki, M. (1995): Sympathetic activity is lower in rats fed a beef tallow diet than in rats fed a safflower oil diet. Metabolism, 44, 934-939.

5) Nicholls, D. G., and Locke, R. M. (1984): Thermogenic mechanisms in brown fat. Physiol. Rev., 64, 1-64.

6) Vander Tuig, J. G., Kerner, J., and Romsos, D. R. (1985): Hypothalamic obesity, brown adipose tissue, and sympathoadrenal activity in rats. Am. J. Physiol., 248, E607-E617.

7) Allars, J., Holt, S. J., and York, D. A. (1987): Energetic efficiency and brown adipose tissue uncoupling protein of obese Zucker rats fed high-carbohydrate and high-fat diets: the effects of adrenalectomy. Int. J. Obese., 11, 591-601.

8) Ricquier, D., Bouillaud, F., Toumelin, P., Mory, G., Bazin, R., Arch, J., and Penicaud, L. (1986): Expression of uncoupling protein mRNA in thermogenic or weakly thermogenic brown adipose tissue. J. Biol. Chem., 261, 13905-13910.

9) Levin, B. E., Triscari, J., and Sullivan, A. C. (1983): Studies of origins of abnormal sympathetic function in obese Zucker rats. Am. J. Physiol., 245, E87-E93.

10) Lin, C., and Klingenberg, M. (1982): Characteristics of the isolated purine nucleotide binding protein from brown fat mitochondria. Biochemistry, 21, 2950-2956.

11) Bradford, M. A. (1976): Rapid and sensitive method for the quantitation of microgram quantities of protein utilizing the principle of proteindye binding. Anal. Biochem., 72, 248-254.

12) Laemmli, U. K. (1970): Cleavage of structual proteins during the assembly of the head of the bacteriophage T4. Nature, 227, 680-685.

13) Burnette, W. N. (1981) "Western blotting": electrophoretic transfer of proteins from sodium dodecyl sulfate-polyacrylamide gels to unmodified nitrocellulose and radiographic detection with antibody and radioiodinated protein A. Anal. Biochem., 112, 195-203.

14) Himms-Hargen, J. (1984): Thermogenesis in brown adipose tissue as energy buffer. N. Engl. J. Med., 311, 1549-1558.

15) Luiten, P. G. M., ter Horst, G. J., and Steffens, A. B. (1987): The hypothalamus: intrinsic connections and outflow pathways to the endocrine system in relation to the control of feeding and metabolism. Prog. Neurobiol., 28, 1-54.

16) Bouillaud, F., Ricqer, D., Mory, G., and Thibault, J. (1984): Increased level of mRNA for the uncoupling protein in brown adipose tissue of rats during thermogenesis induced by cold exposure of norepinephrine infusion. J. Biol. Chem., 259, 
11583-11586.

17) Sadurskis, A., Dicker, A., Cannon, B., and Nedergaard, J. (1995): Polyunsaturated fatty acids recruit brown adipose tissue: increased UCP content and NST capacity. Am. J. Physiol., 269, E351-E360. 